\title{
Clinical manifestations of blood cell parameters and inflammatory factors in 92 patients with COVID-19
}

\author{
Rongrong Deng ${ }^{1 *}$, Changzheng Wang ${ }^{2 \#}$, Yi Ye ${ }^{3}$, Liyao Gou ${ }^{3}, Z_{\text {hongxiao } \mathrm{Fu}^{2}, \mathrm{Bo} \mathrm{Ye}^{3} \text {, Feng Shao }}^{4}$, \\ Xiaomei Zhang ${ }^{3}$, Weiyang $\mathrm{Fu}^{5}$, Jianping Xiao ${ }^{3}$, Tao $\mathrm{Li}^{2}$, Huan $\mathrm{Qi}^{3}$, Chengbin $\mathrm{Li}^{2}$ \\ ${ }^{1}$ Pediatric Medical Center, The Second Clinical Medical College, Yangtze University, Jingzhou, China; ${ }^{2}$ Department of Laboratory Medicine, The \\ Second Clinical Medical College, Yangtze University, Jingzhou, China; ${ }^{3}$ Clinical Department (IVD), Shenzhen Mindray Bio-Medical Electronic \\ Co., Ltd, Shenzhen, China; ${ }^{4}$ Department of Intensive Care Unit(ICU), The Second Clinical Medical College, Yangtze University, Jingzhou, China; \\ ${ }^{5}$ Department of Respiratory Medicine, Jingzhou Infectious Disease Hospital, Jingzhou, China \\ Contributions: (I) Conception and design: C Li, H Qi; (II) Administrative support: Y Ye, L Gou, J Xiao; (III) Provision of study materials or patients: \\ R Deng, C Wang; (IV) Collection and assembly of data: Z Fu, F Shao; (V) Data analysis and interpretation: B Ye, X Zhang; (VI) Manuscript writing: \\ All authors; (VII) Final approval of manuscript: All authors. \\ \#These authors contributed equally to this work. \\ Correspondence to: Professor Chengbin Li. Department of Laboratory Medicine, Jingzhou Central Hospital, The Second Clinical Medical College, \\ Yangtze University, Jingzhou 434023, China. Email: jzlcb002@163.com; Professor Huan Qi, Clinical Department (IVD), Shenzhen Mindray Bio- \\ Medical Electronic Co., Ltd, Shenzhen 518057, China. Email: qihuan@mindray.com.
}

Background: Although there are many COVID-19 case series studies, few studies report the relationship between variations in blood cell parameters and inflammatory factors and disease severity. This study aims to describe the dynamic trends in COVID-19 blood cell parameters and inflammatory factors.

Methods: Ninety-two patients with confirmed COVID-19 at Jingzhou Central Hospital, Hubei Province, China, between January 23, 2020, and April 10, 2020, were enrolled. Epidemiological data, clinical information, and laboratory test results were collected and analyzed.

Results: As patient age increased, disease severity increased $(\mathrm{P}<0.0001)$. The inflammatory factor $\mathrm{C}$-reactive protein (CRP) showed a gradual increasing trend with disease aggravation. Based on dynamic change graphs, CRP in all patients with severe and critical COVID-19 initially increased and then decreased; however, CRP in patients with a good prognosis did not increase again after the initial decline $(<20 \mathrm{mg} / \mathrm{L})$. CRP in patients with a poor prognosis returned to a high level $(>50 \mathrm{mg} / \mathrm{L}) 1$ week after the initial decrease and continued to fluctuate at a high level. Lymphocyte count (LYM\#) in patients with severe and critical disease was significantly lower $\left(<1 \times 10^{9} / \mathrm{L}\right)$ than that in patients with moderate disease; LYM\# was significantly increased 3 weeks after disease onset in patients with a good prognosis $\left(>1 \times 10^{9} / \mathrm{L}\right)$, but patients with a poor prognosis continued to have a low LYM\#.

Conclusions: CRP and LYM\# showed strong correlation with disease progression, suggesting that these parameters could be used to monitor changes in patient condition.

Keywords: COVID-19; SARS-CoV-2; C-reactive protein (CRP); lymphocyte; laboratory

Submitted Nov 09, 2020. Accepted for publication Jan 06, 2021.

doi: 10.21037/atm-20-7765

View this article at: http://dx.doi.org/10.21037/atm-20-7765

(c) Annals of Translational Medicine. All rights reserved. 


\section{Introduction}

In December 2019, an outbreak of pneumonia with an unknown cause occurred, presenting with symptoms of rapidly progressing fever, dry cough, fatigue, muscle pain, and dyspnea (1). Genome sequencing identified the disease pathogen as a novel coronavirus belonging to the $\beta$-coronavirus family, which also includes Middle East respiratory syndrome coronavirus (MERS-CoV) and severe acute respiratory syndrome coronavirus (SARS$\mathrm{CoV})$. The International Committee on Taxonomy of Viruses named the novel coronavirus SARS-CoV-2, with the pneumonia caused by the virus labelled by the World Health organization as 'coronavirus disease 2019', or, more commonly, COVID-19 (2-5). At April 15, 2020, more than 1,980,000 confirmed cases of COVID-19 were recorded worldwide, with a mortality rate of approximately $6.4 \%$ (6).

According to the Novel Coronavirus Pneumonia Diagnosis and Treatment Plan (Provisional $7^{\text {th }}$ Edition) issued by the National Health Commission of the People's Republic of China, COVID-19 is classified into four sub-types: mild, moderate, severe, and critical. Studies report that approximately $80 \%$ of infected patients have mild or moderate cases; however, 26.1-32.0\% of confirmed cases will develop severe or critical disease, with the mortality rate for critical infected patients reported as being up to $61.5 \%$. Therefore, early diagnosis and intervention are key to effective management (7-9). Blood cell analysis is one of the most commonly used laboratory tests in clinical practice and plays an important role in the diagnosis and monitoring of COVID-19. Studies have demonstrated that lymphocyte count (LYM\#) is significantly decreased in severe and critical cases of COVID-19, compared with mild and moderate cases, while neutrophil count (Neu\#) and neutrophillymphocyte ratio (NLR) are significantly increased (10). Changes in the levels of inflammatory factors in blood are also significantly associated with changes in disease status of patients with COVID-19. C-reactive protein (CRP) and procalcitonin (PCT) levels are significantly increased in patients with severe and critical COVID-19 compared with those with mild and moderate disease (11)

Although the measures of blood cells and inflammatory factors are widely used, the usefulness of these parameters in COVID-19, especially dynamic trends with changes in patient condition, is unclear. Without this knowledge, appropriate targeted treatment in clinical practice can be difficult to determine. In this study, laboratory test results of 92 clinically diagnosed and hospitalized COVID-19 patients were analyzed to explore trends in blood cell and inflammatory factors.

We present the following article in accordance with the MDAR reporting checklist (available at http://dx.doi. org/10.21037/atm-20-7765).

\section{Methods}

The study conformed to the provisions of the Declaration of Helsinki (as revised in 2013), and approved by Ethics Committee of Jingzhou Central Hospital. As this study was a retrospective study, there was no patients privacy data such as patient name, ID number, telephone and address were not involved, only demographic information and laboratory testing data of patients were collected and analyzed, so there is no need for informed consent in this study.

\section{Patients}

This study included 92 COVID-19 patients at Jingzhou Central Hospital, 91 of whom had data collected from January 23, 2020 to March 10, 2020, and 1 patient for whom data was collected from January 23, 2020 to April 10, 2020. All infections were confirmed using the new coronavirus COVID-19 nucleic acid detection kit (fluorescence PCR method, Shanghai BioGerm Medical Technology Co., Ltd., China). The classification of patients was based on the Novel Coronavirus Pneumonia Diagnosis and Treatment Plan issued by the National Health Commission of the People's Republic of China (Provisional $7^{\text {th }}$ Edition) (12):

(I) Mild: mild clinical symptoms, no pneumonia manifestations on imaging;

(II) Moderate: fever, respiratory tract and other symptoms, with pneumonia manifestations on imaging;

(III) Severe: adults meeting any of the following criteria: (i) respiratory rate (RR) $\geq 30$ breaths/min; (ii) finger oxygen saturation $\leq 93 \%$ at resting state; (iii) arterial blood partial pressure of oxygen $\left(\mathrm{PaO}_{2}\right)$ /fraction of inspired oxygen $\left(\mathrm{FiO}_{2}\right) \leq 300 \mathrm{mmHg}$; and (iv) lung imaging showing $>50 \%$ lesion progression within 24-48 hours;

(IV) Critical: patients presenting one of the following conditions: (i) respiratory failure and the need for mechanical ventilation; (ii) presence of shock; and (iii) combined with other organ failure requiring intensive care unit (ICU) monitoring and 
treatment.

The most serious disease state that occurred during the course of the disease in each patient was used to determine patient classification. Accordingly, 57 patients presented with moderate disease, 20 patients with severe disease, and 15 patients with critical disease.

\section{Laboratory data collection}

A total of 446 blood cell test results were collected, with at least two test results for each patient. According to the patient's disease classification at the time of blood sample collection, the samples were also divided into three types: moderate, severe, and critical. Among them, 284 cases were moderate, 64 cases were severe, and 97 cases were critical. All blood cell tests were performed using Mindray BC-6800 Auto Hematology Analyzer (Mindray, Shenzhen, China). A total of 514 CRP test results, 127 interleukin 6 (IL-6) test results, and 227 procalcitonin (PCT) test results were collected. Detection of inflammatory factors was performed using AU5800 Biochemical Analyzer (Beckman Coulter, California, US).

\section{Statistical analysis}

All categorical variables are presented as a rate or composition ratio, and all continuous variables are presented as the mean or median. Comparisons of categorical variables were performed using the Pearson's chi-squared test. To reduce the probability of type I error, one-way analysis of variance (ANOVA) was used for comparisons among multiple groups. For pairwise comparisons, if the data generally conformed to a normal distribution, Student's $t$-test was used; otherwise, the data were converted before Student's $t$-test was used. GraphPad Prism Version 8.0 was used for all statistical analyses. $\mathrm{P}<0.05$ was considered statistically significant.

\section{Results}

\section{Presentation characteristics}

As shown in Table 1, a total of 92 hospitalized COVID-19 patients were included in this study: 50 (54.3\%) male patients and $42(45.7 \%)$ female patients. The median age of the patients was 49 years old. There were $42(45.7 \%)$ patients with a history being in Wuhan, 22 (23.9\%) patients with reported close contact with individuals with suspected COVID-19 cases within the previous 2 weeks, and $20(21.7 \%)$ patients with reported close contact with individuals with confirmed COVID-19 cases within the previous 2 weeks. Hypertension [21 (22.8\%)], diabetes [6 $(6.5 \%)]$ and cerebrovascular disease [3 (3.3\%)] were the most common comorbidities. The most common signs at admission were fever [77 (83.7\%)], dry cough [51 (55.4\%)], fatigue [36 (39.1\%)], and chills [26 (28.3\%)]. Some patients also had expectoration [17 (18.5\%)], muscle pain [14 $(15.2 \%)]$, and headache [4 (4.3\%)] at admission.

A comparison between groups showed that patients with critical COVID-19 were older than those with moderate and severe COVID-19. There was no statistically significant difference in the exposure history between patients with different disease severities. The percentage of patients with comorbid hypertension and comorbid malignant tumors was higher in patients with critical disease than in those with severe or moderate disease. There was no statistically significant difference in the signs and clinical manifestations in patients with different disease classifications.

\section{Comparison of blood cell and inflammatory factor detection results between disease classifications}

Comparison of blood parameters showed that with disease aggravation, white blood cell (WBC) and NLR gradually increased, while LYM\# gradually decreased (Figure 1A,B,C). Comparison of inflammatory factors showed that as disease severity increased, CRP expression increased (Figure 1D). The IL-6 level in patients with critical disease was higher than that in patients with moderate disease, but there were no significant differences in IL-6 levels between patients with moderate and severe COVID-19 or between patients with severe and critical COVID-19 (Figure 1E). The expression level of PCT in patients with critical disease was significantly higher than that in patients with severe and moderate disease (Figure $1 F$ ).

\section{Dynamic changes in CRP and LYM\# in patients with different prognoses}

To explore the dynamic trends in CRP and LYM\# in patients with different severity classifications, all patients with severe and critical COVID-19 (27 cases total) with more than three tests were included in the statistics. The 27 patients were divided into two groups. The 'good outcome' group included 19 patients, whose disease was relieved or who recovered and was discharged on the day of sample 
Table 1 Presentation characteristics of COVID-19 patients

\begin{tabular}{|c|c|c|c|c|c|}
\hline Characteristics & Total $(\mathrm{N}=92)$ & Moderate $(\mathrm{N}=57)$ & Severe $(N=20)$ & Critical $(\mathrm{N}=15)$ & $P$ value \\
\hline \multicolumn{6}{|l|}{ Gender } \\
\hline Male & $50(54.3 \%)$ & $29(50.9 \%)$ & $13(65.0 \%)$ & $8(53.3 \%)$ & \multirow[t]{2}{*}{0.551} \\
\hline Female & $42(45.7 \%)$ & $28(49.1 \%)$ & $7(35.0 \%)$ & $7(46.7 \%)$ & \\
\hline Been to Wuhan within 2 weeks & $42(45.7 \%)$ & $28(49.1 \%)$ & $11(55.0 \%)$ & $3(20.0 \%)$ & 0.084 \\
\hline Direct exposure to the Huanan seafood market & $3(3.3 \%)$ & $3(5.3 \%)$ & $0(0 \%)$ & $0(0 \%)$ & 0.386 \\
\hline Close contact with suspected case within previous 2 weeks & $22(23.9 \%)$ & $15(26.3 \%)$ & $3(15.0 \%)$ & $4(26.7 \%)$ & 0.572 \\
\hline Close contact with confirmed case within previous 2 weeks & $20(21.7 \%)$ & $13(22.8 \%)$ & $4(20.0 \%)$ & $3(20.0 \%)$ & 0.951 \\
\hline Cardiovascular disease & 0 & 0 & 0 & 0 & \\
\hline Diabetes & $6(6.5 \%)$ & $3(5.3 \%)$ & 0 & $3(20.0 \%)$ & 0.050 \\
\hline Malignancy & $2(2.2 \%)$ & 0 & 0 & $2(13.3 \%)$ & $0.005^{\mathrm{b}}$ \\
\hline Cerebrovascular disease & $3(3.3 \%)$ & $1(1.8 \%)$ & $1(5.0 \%)$ & $1(6.7 \%)$ & 0.562 \\
\hline COPD & 0 & 0 & 0 & 0 & \\
\hline Chronic kidney disease & $1(1.1 \%)$ & 0 & 0 & $1(6.7 \%)$ & 0.075 \\
\hline Viral hepatitis & $1(1.1 \%)$ & $1(1.8 \%)$ & 0 & 0 & 0.733 \\
\hline \multicolumn{6}{|l|}{ Signs and symptoms } \\
\hline Myalgia & $14(15.2 \%)$ & $12(21.1 \%)$ & $2(10.0 \%)$ & 0 & 0.099 \\
\hline Headache & $4(4.3 \%)$ & $4(7.0 \%)$ & 0 & 0 & 0.277 \\
\hline
\end{tabular}

$P$ value indicates differences between moderate, severe and critical. $\mathrm{P}<0.05$ was considered statistically significant. ${ }^{a}$, severe vs. critical, $\mathrm{P}<0.05$; ${ }^{b}$, moderate vs. critical, $\mathrm{P}<0.05$. COPD, chronic obstructive pulmonary disease.

collection. The 'poor outcome' group included 8 patients whose disease remained critical or who died on the day of sample collection.

The graph for CRP dynamic changes showed that within the first 2 weeks of onset, CRP in the two groups of patients initially increased and then decreased; however, 4 weeks after onset, CRP in the 'poor outcome' group significantly increased again and continued to fluctuate at a high level $(>50 \mathrm{mg} / \mathrm{L})$, whereas CRP in the 'good outcome' group did not increase again (Figure 2A). To control for the influence of individual differences and treatment differences, the graphs for dynamic changes in the two groups of patients were aligned by taking the first CRP peak as the reference point. The results showed that almost all patients showed an initial increase in CRP with subsequent decrease; however, CRP in patients in the 'poor outcome' group significantly increased again 1 week after decreasing $(>50 \mathrm{mg} / \mathrm{L})$, while CRP in patients in the 'good outcome' group continued 
A
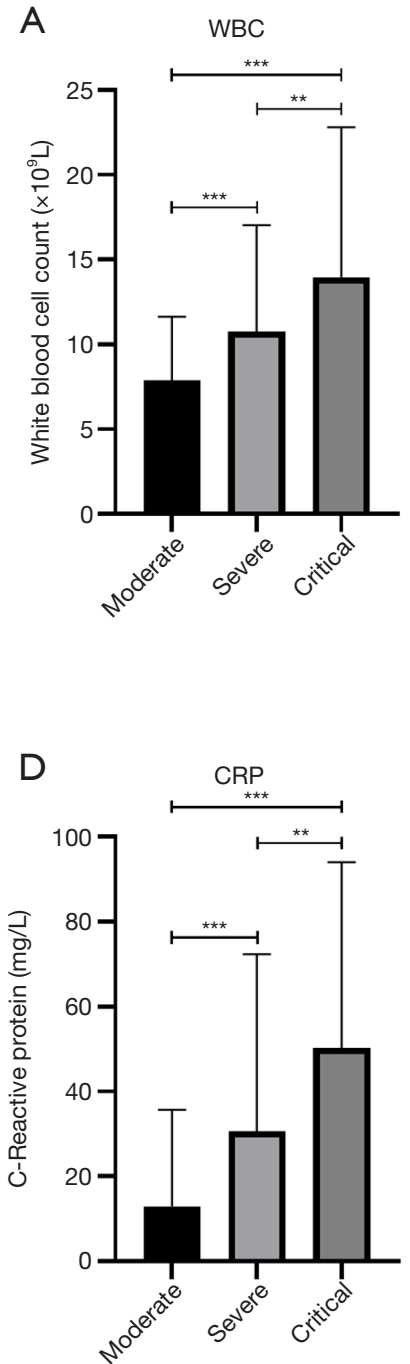

B

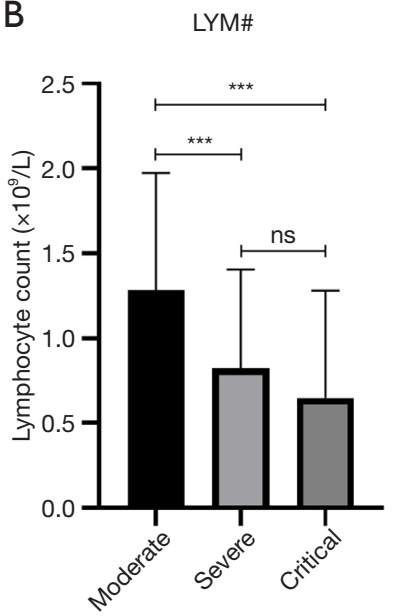

E

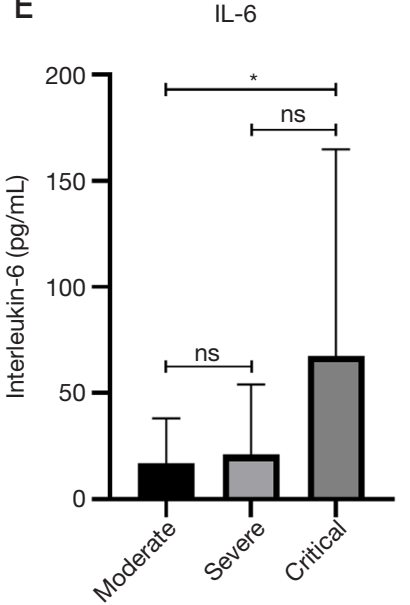

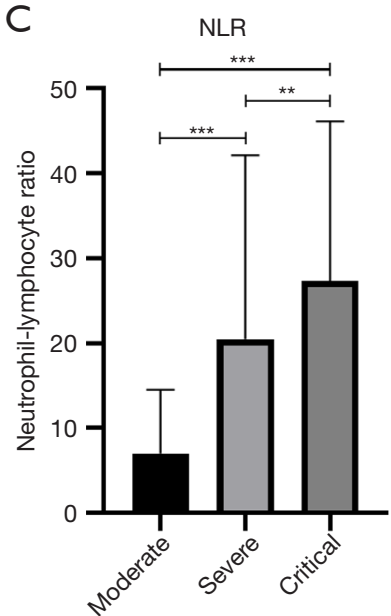

F $\quad$ PCT

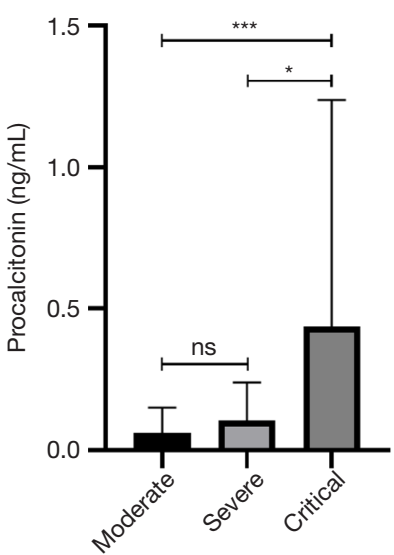

Figure 1 Comparison of blood cell parameters and inflammatory factors in patients with different severities of COVID-19. Comparison of the blood cell and inflammatory factor detection results in patients with different disease severities. Data are shown as the mean $\pm \mathrm{SD}$. ***, $\mathrm{P}<0.001$; **, $\mathrm{P}<0.01$; *, $\mathrm{P}<0.05$. WBC, white blood cell; LYM\#, lymphocyte count; NLR, neutrophil-lymphocyte ratio; CRP, C-reactive protein; IL-6, interleukin-6; PCT, procalcitonin; ns, non-significant.

to stay at a low level after the initial decrease $(<20 \mathrm{mg} / \mathrm{L})$ (Figure $2 B$ ). Analysis of dynamic trends in LYM\# showed that LYM\# was significantly lower at onset $\left(<1 \times 10^{9} / \mathrm{L}\right)$. LYM\# significantly increased 3 weeks after onset or 1 week after the first CRP peak $\left(>1 \times 10^{9} / \mathrm{L}\right)$ in patients with a good outcome, whereas LYM\# in patients with a poor outcome remained at a low level (Figure 2C,D).

A comparison between the two groups of CRP levels at $10-15$ days and at 28 days or longer post-onset showed that CRP in patients with a good outcome significantly decreased at 28 days or longer after onset, while CRP in patients with a poor outcome significantly increased 28 days or longer after onset (Figure 2E). The LYM\# results were compared at 7-12 days after onset and 21 days or longer after onset between the two groups of patients. The results showed that the LYM\# in the patients with a good outcome increased significantly at 21 days or longer after onset, while the LYM\# in the patients with a poor outcome did not change significantly 21 days or longer after onset (Figure 2F). 

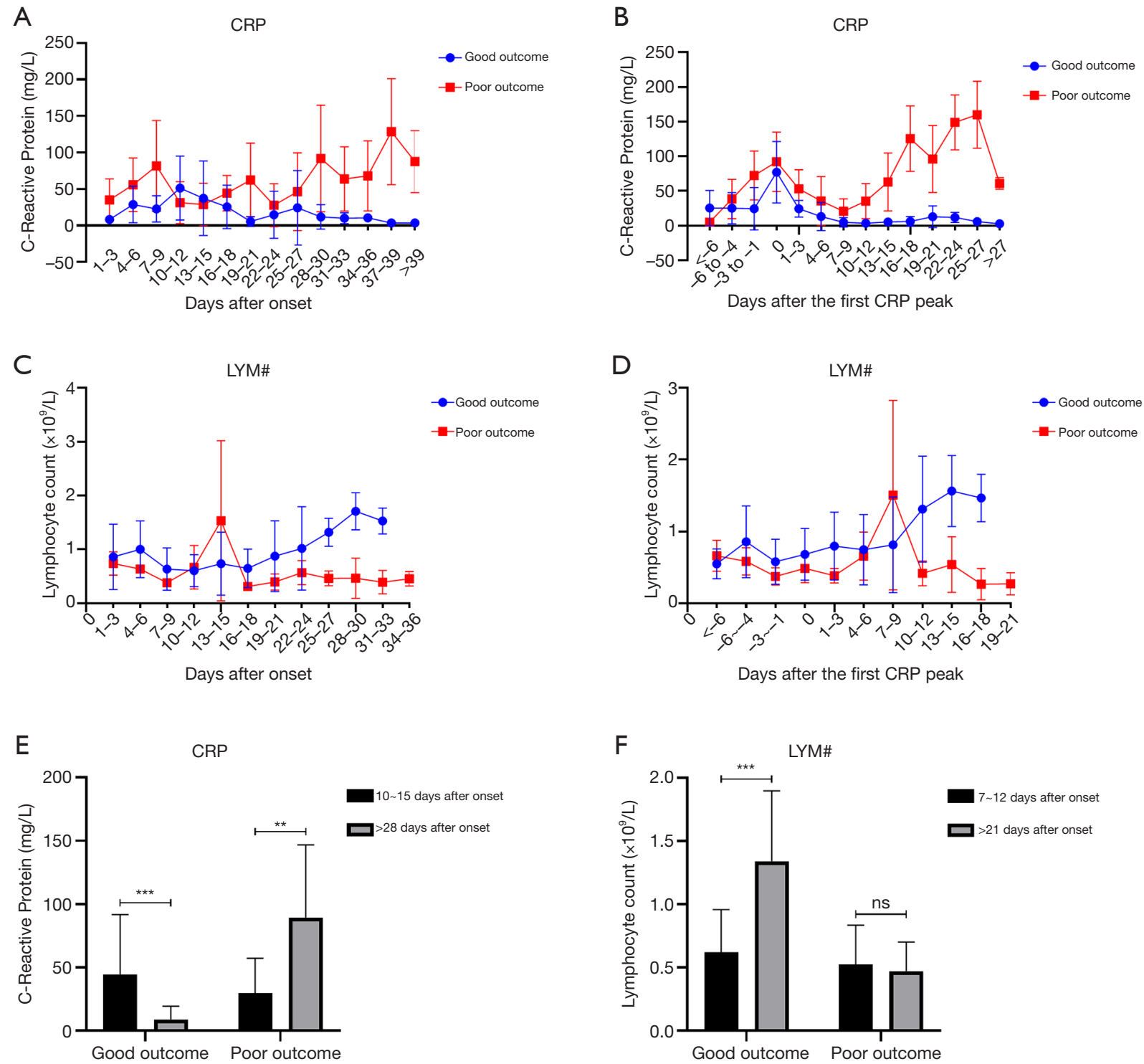

Figure 2 Dynamic changes in CRP and LYM\# in patients with different outcomes. The graphs for the dynamic changes of CRP (A,B) and LYM\# (C,D) in 27 patients (19 with a poor prognosis and 8 with a good prognosis). The histograms show the differences in the CRP and LYM\# results between the two groups of patients at different time periods of disease progression $(\mathrm{E}, \mathrm{F})$. Data are shown as the mean $\pm \mathrm{SD}$. ***, $\mathrm{P}<0.001 ;{ }^{* *}, \mathrm{P}<0.01$. ns, non-significant.

\section{Case analysis of CRP dynamic change}

Figure 3 shows trends in CRP across disease progression in a patient with critical COVID-19. Based on etiology and other laboratory test results, the disease condition was divided into five stages: (I) pulmonary viral infection; (II) pulmonary bacterial infection; (III) pulmonary fungal infection; (IV) urinary tract infection; and (V) bloodstream bacterial infection. The name of each stage denotes the dominant type of infection, but does not exclude the possibility of other types of infection at that stage.

In the first stage, at 11 days after disease onset, pulmonary viral infection lesions expanded, and CRP increased accordingly. After an increase in the dose of glucocorticoids, the infection was controlled, and CRP decreased accordingly. In the second stage, bacterial infection occurred in the lungs, and CRP increased sharply. Advanced antibacterial drugs were used to control bacterial 


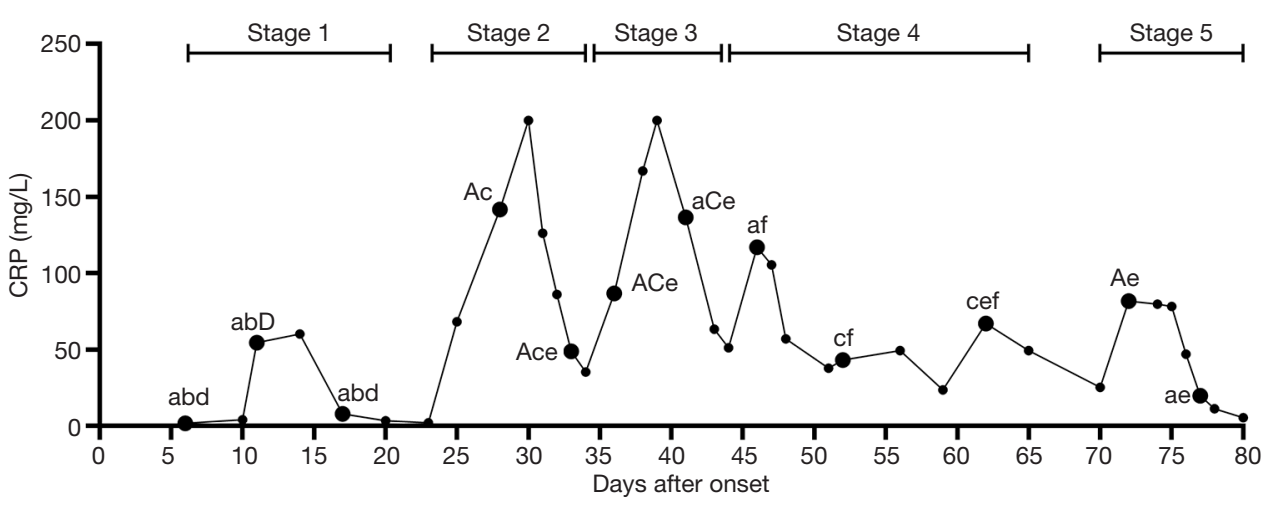

Figure 3 CRP dynamic changes in a patient. The dynamic changes of CRP with disease progression in a patient. a, antibacterial drugs; A, advanced antibacterial drugs; b, antivirus drugs; c, antifungal drugs; C, advanced antifungal drugs; d, glucocorticoids; D, high-dose glucocorticoids; e, sputum suction by fiber bronchoscope; f, urinary tract flushing.

infections, and antifungal drugs were used in combination to prevent fungal infections. After bacterial infections were controlled, CRP also decreased. In the third stage, fungal infection occurred in the lungs, and CRP was significantly increased. After treatment with advanced antifungal for fungal infections, combined with the use of fiberoptic bronchoscopy for sputum suction, fungal infections were controlled, and CRP levels decreased accordingly. In the fourth stage, the patient had a urinary tract infection, and CRP increased accordingly. After treatment with antibacterial drugs, antifungal drugs and continuous urinary tract flushing, the infections were controlled, and CRP decreased accordingly. In the fifth stage, the patient had a bloodstream infection, and CRP increased accordingly. After advanced antibiotic treatment, the infections were controlled, and the CRP decreased accordingly.

\section{Discussion}

At present, many large-sample case series analyses exist with complete COVID-19 records, and characteristics of laboratory test results of COVID-19 patients have been reported; however, there are few studies that report dynamic trends of blood cell parameters and inflammatory factors with disease progression. In addition, as the mechanism of changes in blood cell parameters and inflammatory factors in COVID-19 is not yet clear, the use of these parameters to guide clinical interventions is still controversial $(13,14)$. This report demonstrated that blood cell parameterssuch as WBC, LYM\#, and NLR - as well as inflammatory factors - such as CRP, IL-6, and PCT-showed changes in trends with disease progression, indicating that blood cell parameters and inflammatory factors can be used to guide clinical treatment.

This report included 92 patients with COVID-19 recruited between January 23, 2020 and April 10, 2020, at Jingzhou Central Hospital, Hubei Province. Of these, 57 patients were classified as moderate severity, 20 patients as severe, and 15 patients as critical. Epidemiological and clinical information showed that elderly patients were more seriously ill $(\mathrm{P}<0.0001)$, which is consistent with the results of other case series analysis reports (15), suggesting that the progression of COVID-19 is age-related. There was no statistically significant correlation between exposure history in the epidemic origin and disease severity, nor was contact history of infected person $(\mathrm{P}>0.05)$. The percentages of patients with critical disease with comorbid hypertension and comorbid malignant tumors were relatively high $(\mathrm{P}<0.05)$, indicating that patients with these underlying diseases often present with a more serious presentation, requiring the attention of clinicians. Contrary to the results of other studies, the differences in the common signs and symptoms of patients with different severity levels were not statistically significant (16). This may be because the patient information in this report was collected at admission when most patients were at an early stage of the disease.

In almost all other research reports, only one laboratory test result was included for comparison for each patient. This test result could have been collected at the time of admission, exacerbation, or discharge. Furthermore, this method of comparison may conceal changes in the parameters of a single patient when the patient's condition changes. Therefore, in this report, the samples were categorized according to disease severity at the time 
of blood sample collection, and then all samples were compared. The advantage of this comparison method is that the results cover the differences in the results for different patients and the differences in the results at different stages for the same patient; however, this method of comparison also has a major drawback, and that is that different patients may have large sample size deviations, resulting in biased results. Therefore, this report only included patients who had at least three blood cell test results or inflammatory factor test results for comparison, aimed to balance the number of samples for each patient. The results showed that WBC and NLR significantly increased with disease aggravation, while LYM\# significantly decreased, indicating that the proliferation of the bone marrow granulocyte system was active, the lymphatic system was inhibited, or lymphocytes were damaged and phagocytosed, which is consistent with the results reported in other studies. The comparison of inflammatory factors showed that CRP significantly increased with disease severity. Interestingly, there was no significant difference in IL-6 and PCT results between patients with moderate and severe COVID-19, and the expression levels of IL-6 and PCT in patients with critical COVID-19 were significantly increased. The trend differences among CRP, IL-6 and PCT may reflect the time dependence of the regulation of inflammatory factor expression; however, this reasoning require further studies for confirmation.

To explore different trends in blood cell parameters and inflammatory factors in patients with severe and critical COVID-19 with different prognoses, this study plotted dynamic change graphs for CRP and LYM\# in patients who had different clinical outcomes as the disease progressed. The graph for the dynamic change in CRP showed that almost all patients exhibited a CRP trend of an initial increase and subsequent decrease during the course of disease development; however, due to differences in treatment methods and responses to treatment, the time points of the increase and decrease in CRP in each patient were different, which may have obscured the differences in CRP in patients with different prognoses. Therefore, in this study, the first CRP peak was used as the reference point, and the number of days from the first CRP peak was used as the $\mathrm{x}$-axis to plot dynamic changes. The results showed that in almost all patients, CRP decreased to a low level $(<20 \mathrm{mg} / \mathrm{L}) 1$ week after CRP first peaked; however, in patients with a good outcome, CRP did not increase again after the decrease, but in patients with a poor outcome, CRP increased significantly after the decrease $(>50 \mathrm{mg} / \mathrm{L})$, which may indicate aggravated lung infection or the occurrence of other organ infection, resulting in poor patient prognosis. The graphs for the dynamic trends in LYM\# showed that LYM\# was significantly lower at disease onset in patients with severe and critical disease $\left(<1 \times 10^{9} / \mathrm{L}\right)$; LYM\# significantly increased again 3 weeks after onset in patients with a good outcome $\left(>1 \times 10^{9} / \mathrm{L}\right)$, and LYM\# remained at a low level in patients with a poor outcome, indicating that the second increase in LYM\# may reflect the effectiveness of antiviral treatment. Notably, an outlier was observed in the dynamic change graphs for LYM\#. The LYM\# in the poor prognosis group increased sharply at 13 to 15 days after onset and then decreased sharply. This is because the LYM\# increased to as high as $4.10 \times 10^{9} / \mathrm{L}$ and $\mathrm{WBC}$ increased to $54.65 \times 10^{9} / \mathrm{L}$ in an 81 -year-old male patient. It is possible that this patient had concurrent bacterial infection and/or other viral infections.

The case analysis of the dynamic changes showed CRP trends in a critical COVID-19 patient. The result suggested that at all stages of disease development in the patient, CRP significantly increased initially. After the use of drugs, or the lesion was cleared to control the infection, CRP decreased accordingly. This case analysis showed that CRP can be used to monitor COVID-19 severity, coinfection of other pathogens and infection control effects; however, because only one case with complete information was presented, this result may have poor generalizability. In subsequent studies, more case studies and better data visualization is necessary.

This study presented clinical information, blood cell parameters, and inflammatory factors for 92 patients with COVID-19, and explored the dynamic trends in these measures with disease progression. The analysis indicated that laboratory test results-such as CRP and LYM\#can be used to monitor change in a patient's condition. Our future research will be a multi-site study involving the collection of more general information and laboratory test results for COVID-19 and will involve continuous observation of the relationship between changes in disease status and laboratory test results, to clarify the test efficacy of CRP and LYM\# as predicting factors for disease changes and prognosis.

\section{Acknowledgments}

Thank you to all people who work so hard to fight against COVID-19.

Funding: None. 


\section{Footnote}

Reporting Checklist: The authors have completed the MDAR reporting checklist. Available at http://dx.doi.org/10.21037/ atm-20-7765

Data Sharing Statement: Available at http://dx.doi. org/10.21037/atm-20-7765

Conflicts of Interest: All authors have completed the ICMJE uniform disclosure form (available at http://dx.doi. org/10.21037/atm-20-7765). The authors have no conflicts of interest to declare.

Ethical Statement: The authors are accountable for all aspects of the work in ensuring that questions related to the accuracy or integrity of any part of the work are appropriately investigated and resolved. The study conformed to the provisions of the Declaration of Helsinki (as revised in 2013), and approved by Ethics Committee of Jingzhou Central Hospital. As this study was a retrospective study, there was no patients privacy data such as patient name, ID number, telephone and address were not involved, only demographic information and laboratory testing data of patients were collected and analyzed, so there is no need for informed consent in this study.

Open Access Statement: This is an Open Access article distributed in accordance with the Creative Commons Attribution-NonCommercial-NoDerivs 4.0 International License (CC BY-NC-ND 4.0), which permits the noncommercial replication and distribution of the article with the strict proviso that no changes or edits are made and the original work is properly cited (including links to both the formal publication through the relevant DOI and the license). See: https://creativecommons.org/licenses/by-nc-nd/4.0/.

\section{References}

1. Zhu N, Zhang D, Wang W, et al. A Novel Coronavirus from Patients with Pneumonia in China, 2019. N Engl J Med 2020;382:727-33.

2. Chan JF, Yuan S, Kok KH, et al. A familial cluster of pneumonia associated with the 2019 novel coronavirus indicating person-to-person transmission: a study of a family cluster. Lancet 2020;395:514-23.

3. Lu R, Zhao X, Li J, et al. Genomic characterisation and epidemiology of 2019 novel coronavirus: implications for virus origins and receptor binding. Lancet 2020;395:565-74.

4. Chan JF, Kok KH, Zhu Z, et al. Genomic characterization of the 2019 novel human-pathogenic coronavirus isolated from a patient with atypical pneumonia after visiting Wuhan. Emerg Microbes Infect 2020;9:221-36.

5. Coronaviridae Study Group of the International Committee on Taxonomy of Viruses. The species Severe acute respiratory syndrome-related coronavirus: classifying 2019-nCoV and naming it SARS-CoV-2. Nat Microbiol 2020;5:536-44.

6. COVID-19 Dashboard by the Center for Systems Science and Engineering (CSSE) at Johns Hopkins University (JHU). Available online: https://coronavirus.jhu.edu/map. html, accessed 15 April 2020.

7. Huang C, Wang Y, Li X, et al. Clinical features of patients infected with 2019 novel coronavirus in Wuhan, China. Lancet 2020;395:497-506.

8. Yang X, Yu Y, Xu J, et al. Clinical course and outcomes of critically ill patients with SARS-CoV-2 pneumonia in Wuhan, China: a single-centered, retrospective, observational study. Lancet Respir Med 2020;8:475-81.

9. Zhou H, Xu K, Shen Y, et al. Coronavirus disease 2019 (COVID-19): chest CT characteristics benefit to early disease recognition and patient classification-a single center experience. Ann Transl Med 2020;8:679.

10. Liu J, Liu Y, Xiang P, et al. Neutrophil-to-Lymphocyte Ratio Predicts Severe Illness Patients with 2019 Novel Coronavirus in the Early Stage. medRxiv (Preprint). 2020.02.10.20021584. Available online: https://doi. org/10.1101/2020.02..20021584.

11. Guan WJ, Ni ZY, Hu Y, et al. Clinical Characteristics of Coronavirus Disease 2019 in China. N Engl J Med 2020;382:1708-20.

12. China National Health Commission. Diagnosis and treatment of pneumonia caused by new coronavirus infection (version 7) (Mar 3, 2020). Beijing: China National Health Commission, 2020.

13. Chen N, Zhou M, Dong X, et al. Epidemiological and clinical characteristics of 99 cases of 2019 novel coronavirus pneumonia in Wuhan, China: a descriptive study. Lancet 2020;395:507-13.

14. Mardani R, Ahmadi Vasmehjani A, Zali F, et al. Laboratory Parameters in Detection of COVID-19 Patients with Positive RT-PCR; a Diagnostic Accuracy Study. Arch Acad Emerg Med 2020;8:e43.

15. Wang $\mathrm{M}, \mathrm{Wu} \mathrm{Q}, \mathrm{Xu} \mathrm{W}$, et al. Clinical diagnosis of 8274 
Page 10 of 10

samples with 2019-novel coronavirus in Wuhan. medRxiv (Preprint). 2020.02.12.20022327. Available online: https:// doi.org/10.1101/2020.02.12.20022327

16. Wang D, Hu B, Hu C, et al. Clinical Characteristics of 138 Hospitalized Patients With 2019 Novel Coronavirus-

Cite this article as: Deng R, Wang C, Ye Y, Gou L, Fu Z, Ye B, Shao F, Zhang X, Fu W, Xiao J, Li T, Qi H, Li C. Clinical manifestations of blood cell parameters and inflammatory factors in 92 patients with COVID-19. Ann Transl Med 2021;9(1):62. doi: 10.21037/atm-20-7765

\section{Deng et al. Dynamic change of laboratory tests of COVID-19 patients}

Infected Pneumonia in Wuhan, China. JAMA 2020;323:1061-9.

(English Language Editor: M. Hawkins) 